\title{
Transoral laser microsurgery in early glottic carcinoma: an Egyptian experience
}

\author{
Amr El-Badrawy ${ }^{1}$, Mohamed Amin ${ }^{1,2}$, Shreif Bahaa Elden ${ }^{3}$ and Ahmed Yousef ${ }^{1 *}$ (D)
}

\begin{abstract}
Background: This is a prospective study evaluating oncologic outcomes of early glottic carcinoma treated with transoral laser micro-laryngeal (TLM) surgery in two tertiary referral hospital. Patients who underwent TLM for early glottic carcinoma at the Otolaryngology Department and National Cancer Institute-Cairo University during the period between January 2016 and July 2019 who met inclusion criteria were included in the study. Operative and postoperative complications were assessed. Patients were followed up to assess loco-regional control.

Results: Thirty-three patients were included in the study. There were no operative complications. Median postoperative pain score was 3. Median duration of procedure was $15 \mathrm{~min}$. None of the patients experienced postoperative bleeding or airway complications. There was no return to hospital within 30 days for complications. Median follow-up was 24 months. Disease-free survival was 97\%. Overall survival was 100\%.

Conclusion: Transoral laser microsurgery is a secure and successful choice for the treatment of glottic carcinomas, related to negligible morbidity and great local control.
\end{abstract}

Keywords: Early glottic carcinoma, TLM, Follow-up, Recurrence

\section{Background}

Laryngeal carcinoma is a common neoplasm representing $1 \%$ of all malignancies diagnosed annually and about $35 \%$ of all head and neck malignancies.

Ninety percent of the malignant tumors of the larynx are squamous cell carcinomas, with distinctive distributions of predominance based on the particular subsite influenced (glottic, supraglottic, and subglottic) [1].

Glottic carcinoma is the most common representing $70 \%$ of laryngeal cancers and usually presents early with hoarseness.

Laryngeal carcinoma is usually suspected when a circumscribed area of mucosal change is discovered, for example, leukoplakia which is common in the middle of the mobile part of the vocal cord.

\footnotetext{
* Correspondence: Drahmedyousef2012@yahoo.com

'Otolaryngology Department, Kasr Al-Aini, Faculty of Medicine, Cairo

University, Cairo, Egypt

Full list of author information is available at the end of the article
}

Suspicion of carcinoma in situ, micro-invasion, or frank carcinoma (microcarcinoma) is confirmed histopathologically by a biopsy [2].

Fortunately, indeed the earliest glottic cancer changes the voice by influencing wave pattern forms over the vocal fold. Since ordinary voice generation depends on the integrity of a six-cell thick epithelium and a sensitive jelly-like shallow lamina propria, indeed carcinoma in situ may create significant voice alter. Thus, any person with change of voice quality persisting for 3 weeks or more should be referred urgently to an otolaryngologist to exclude malignancy.

For supraglottic tumors, long delays within the introduction to essential care are common due to the unclearness of symptoms such as "globus" and otalgia, and thus, numerous are progressed and have a nodal illness at the time of introduction [3].

Consequently, if we could effectively deal with glottic cancer early, we would have great outcomes in management of laryngeal carcinomas generally and avoid the hazardous complications in advanced stages.

\section{Springer Open}

(c) The Author(s). 2021 Open Access This article is licensed under a Creative Commons Attribution 4.0 International License, which permits use, sharing, adaptation, distribution and reproduction in any medium or format, as long as you give appropriate credit to the original author(s) and the source, provide a link to the Creative Commons licence, and indicate if changes were made. The images or other third party material in this article are included in the article's Creative Commons licence, unless indicated otherwise in a credit line to the material. If material is not included in the article's Creative Commons licence and your intended use is not permitted by statutory regulation or exceeds the permitted use, you will need to obtain permission directly from the copyright holder. To view a copy of this licence, visit http://creativecommons.org/licenses/by/4.0/. 
This is what we evaluated in our study and assessed the role of $\mathrm{CO} 2$ laser in this manner.

\section{Methods}

Between the dates of January 2016 and July 2019, 33 patients with early-stage glottic carcinoma underwent transoral laser microsurgery (TLM) at the Otorhinolaryngology (ORL) Department of Faculty of Medicine and Cairo University and National Cancer Institute (NCI). The study was approved by scientific committee of ORL Department of Cairo University and ethics committee of Faculty of Medicine Cairo University, but the committee reference number is not available. The informed written consent was obtained from all patients.

\section{Patient selection}

The patients who were included in the study have glottic carcinoma in situ, T1a, or T1b. The patients with a previous history of glottic cancer or local recurrence after radiotherapy were excluded. All patients were smokers as a risk factor. The 2017 American Joint Committee on Cancer (AJCC) was used to stage lesions [4].

\section{Inclusion criteria}

- Patient with T (Is-1-2), N0, M0 Cancer Larynx

- Direct laryngoscopy DL and biopsy reveals squamous cell carcinoma or carcinoma in situ

\section{Exclusion criteria}

- Patients who refused to consent for enrollment for surgery and its complication like tracheostomy

- Patients who were unfit for general anesthesia

- Patients with lesion reaching the anterior commissure
- Patients with involvement of the paraglottic spaces

- Patients with involvement of the cricoarytenoid joint

- Patients with local recurrence after radiotherapy

\section{Preoperative}

A detailed history was taken including a history of hoarseness, dysphagia, odynophagia, neck mass, dyspnea, referred otalgia, and aspiration which are symptoms consistent with laryngeal carcinoma, besides a history of previous intubation and stridor. Full otorhinolaryngology, head and neck examination including flexible laryngoscope was fulfilled. All patients underwent preoperative assessment including laboratory investigations $(\mathrm{CBC}$, Coagulation profile, renal and liver function tests). Radiological assessment of head and neck (CT with contrast) if needed. Direct laryngoscope with a microscope for definite mapping of lesion and biopsy was performed.

\section{Technique}

- Procedures were done under general anesthesia with patient in supine position.

- General anesthesia with endotracheal intubation size 5-mm inner diameter causes little obstruction of operative field.

- The cuff of tube was filled with saline, protected with saline-soaked swabs and placing additional moistened swabs distal to the lesion to be operated on.

- Direct laryngoscopy was introduced transorally into larynx and supported on the platform above the patient's chest as in Fig. 1.

- The patient's face and particularly the eyes should be covered with a moistened towel.

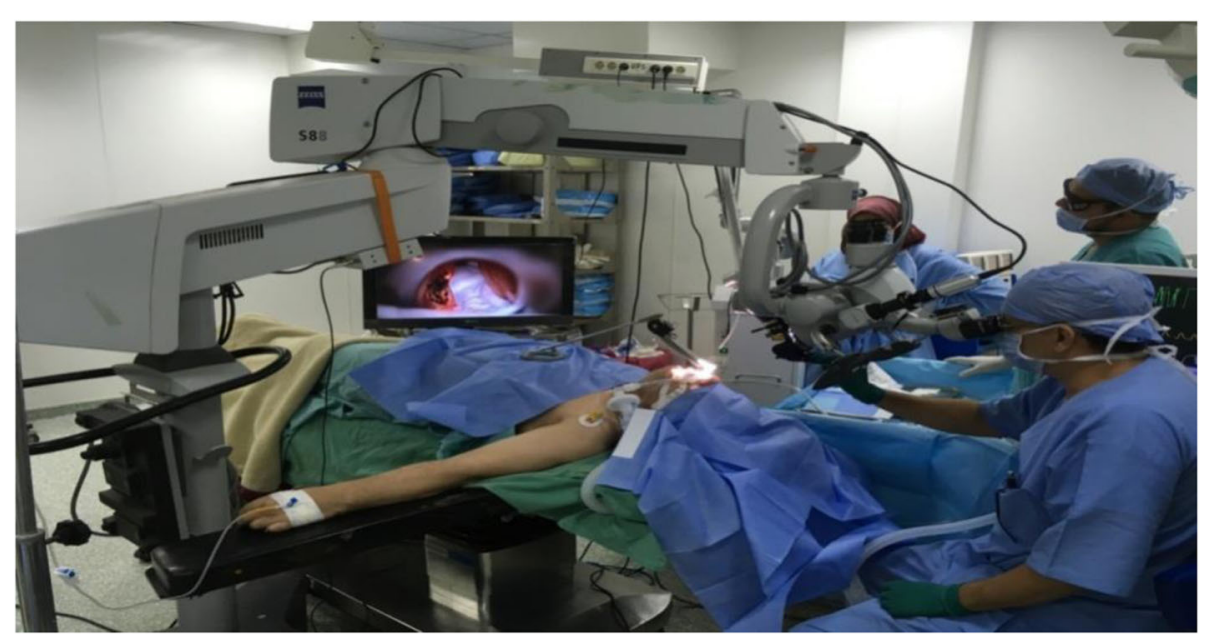

Fig. 1 Showing set up during surgery 


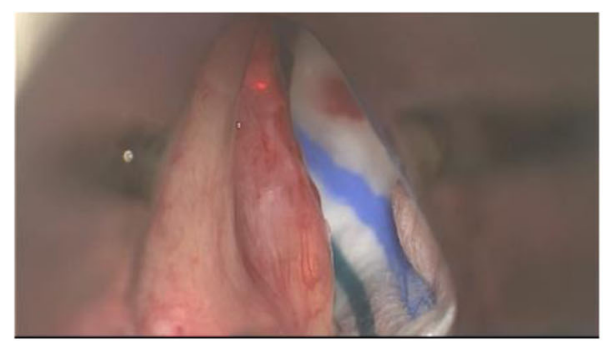

(A)

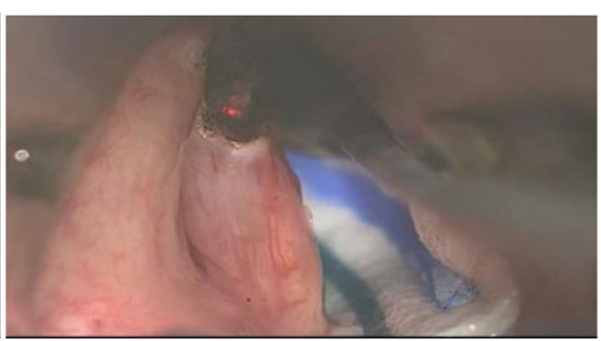

(B)

Fig. 2 a Intraoperative view of a T1a carcinoma of the right vocal cord before laser microsurgical resection and $\mathbf{b}$ immediately after resection

- The excision was performed using a CO2 LASER by a Zeiss surgical microscope (model S88-73446; Carl Zeiss, oberkochen, Germany) and laser setting was super pulse mode and 8-10 W for power.

- The micromanipulator was manipulated by the right hand, while the left hand was holding a grasping forceps introduced through the laryngoscope.

- Backstop micro-instrument with suction channel for evacuation of vapor and protecting the mucosa against accidental laser-beam exposure.

- Both cutting and coagulation properties of the laser were used according to the lesion with proper safety margin as shown in Fig. 2.

- Removing any char formed on the cut surface, by wiping it with a moist swab.

- The attached video (movie1) shows our technique in excision of glottic lesions.

\section{Postoperative}

Visual analog scale (VAS) was used directly after the operation to assess the postoperative pain and determine the severity of pain numerical as in Fig. 3 [5].

The patients were usually discharged from the hospital on the first day or second day of surgery. The patients were advised to relax the voice for 2 weeks and to avoid smoking. Anti-reflux medications, anti-edematous, and low dose steroids were prescribed for all patients.

Follow-up examinations were performed every month for the first 6 months postoperative and every 3 months for the next year to exclude recurrence as shown in Fig. 4.

\section{Voice Handicap Index (VHI-10) scores}

Subjective voice assessment was performed for all patients by postoperative VHI-10 questionnaires. The questionnaire is composed of 30 questions that cover 3 aspects: functional, physical, and emotional. Each question is given score between 0 (lower disability) and 4 (higher disability). The total VHI score ranges from 0 to 120 , as 120 is the most severe voice problem. The voice assessment was performed after 6 months of the operation [6].

\section{Data management and analysis}

Data were coded and entered using the statistical package SPSS (Statistical Package for the Social Sciences) version 25. Data were summarized using mean, standard deviation, median, minimum and maximum in quantitative data, and frequency (count) and relative frequency (percentage) for categorical data.

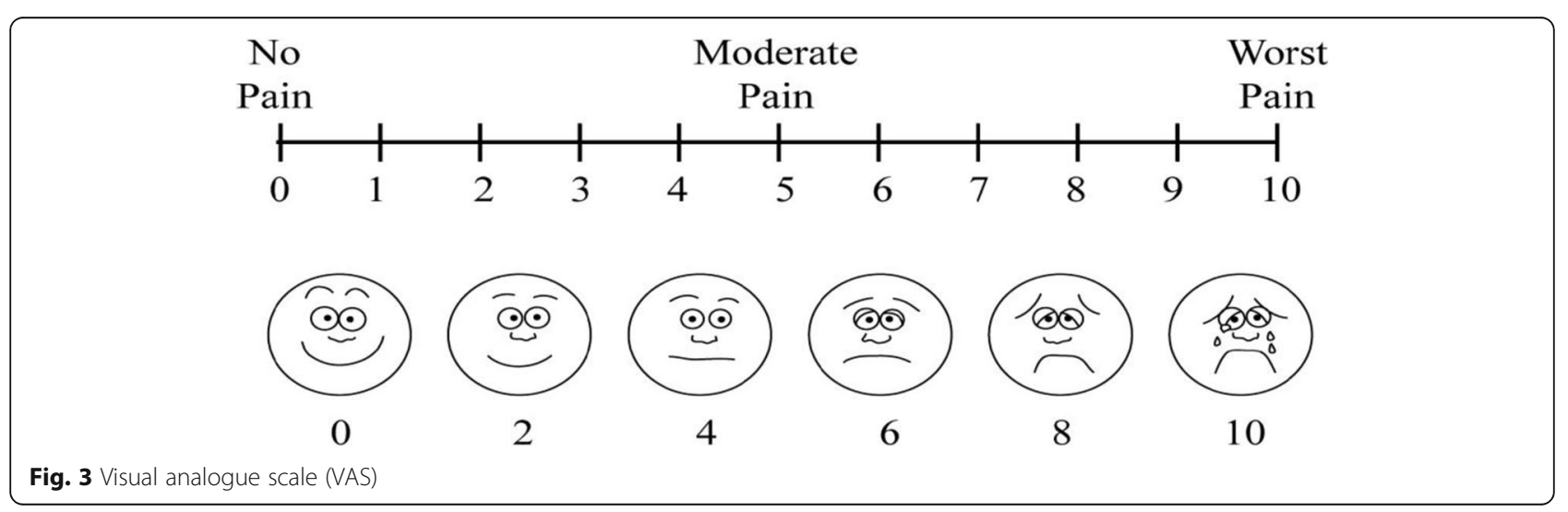




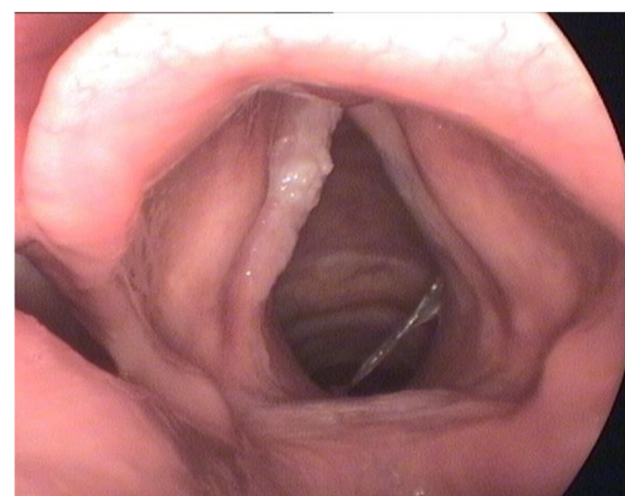

(A)

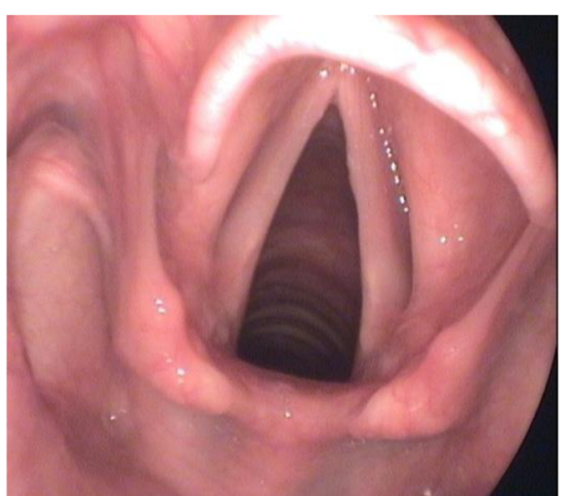

(B)

Fig. 4 a Left vocal cord lesion. b Six months postoperative laser excision

\section{Results}

\section{Description of our sample \\ Age}

The age of our patients ranges from 37 to 80 years old and the mean age was $60.54 \pm 10.06$ as shown in Table 1 .

\section{Sex}

The patients in our study were 32 males and 1 female.

\section{Presenting symptom}

All patients presented with hoarseness of voice.

\section{Site of lesion}

In our study, 18 patients had right glottic lesion, 11 patients had left glottic lesion, and 4 patients had bilateral lesions as illustrated in Fig. 5.

\section{Comorbidities}

In this study, one patient had hepatitis $C$, one patient had controlled diabetes, 2 patients had hypertension, and the rest of patients did not have any medical problems as shown in Table 2.

\section{Staging of lesion}

Of 33 patients, $33.3 \%$ had carcinoma in situ Tis $(n=11)$, $54.6 \%$ had T1a $(n=18)$, and $12.1 \%$ had T1b $(n=4)$.

The pathology of the lesion in 22 patients $(66 \%)$ was squamous cell carcinoma; 11patients (33.3\%) had carcinoma in situ with high-grade dysplasia.

Table 1 Descriptive statistics of age

\begin{tabular}{llllll}
\hline & Mean & Standard deviation & Median & Minimum & Maximum \\
\hline Age & 60.54 & 10.06 & 59.00 & 37.00 & 80.00 \\
\hline
\end{tabular}

\section{Primary outcomes}

Of the 33 patients treated by CO2 LASER and through 24 months' follow-up, 31 patients (94\%) had no recurrence after treatment.

In one patient, total resection could not be achieved and referred to radiotherapy. Another patient had recurrence after 5 months from surgery as illustrated in Fig. 6.

\section{Duration of procedure}

The duration of transoral laser microsurgery (TLM) ranges from 10 to $40 \mathrm{~min}$ and mean procedure time was17.75 \pm 7.12 $\mathrm{min}$ as illustrated in Table 3.

\section{Postoperative pain}

According to the visual analog scale (VAS) of pain, the postoperative pain ranges from 2 to 5 as shown in Table 3.

\section{Voice assessment}

According to VHI Score, 12 patients (36.4\%) had mild voice disability (under 30 points), 20 patients (60.6\%) had moderate voice disability (31-60 points), and one patient had severe disability (61-90 points).

\section{Complications}

No complications regarding the procedure were recorded. Tracheostomy was not needed in any of the patients. They did not suffer from symptoms of, dyspnea, severe pain, hemoptysis, bleeding, surgical site infection, and other serious complications.

\section{Discussion}

The main treatment modalities for early laryngeal carcinomas are organ sparing surgery in the form of transoral laser microsurgery (TLM), open partial laryngectomy (OPL), and radiotherapy (RT).

They are all used as primary treatment and the treatment of recurrent diseases [7]. 


\section{Site of lesion}

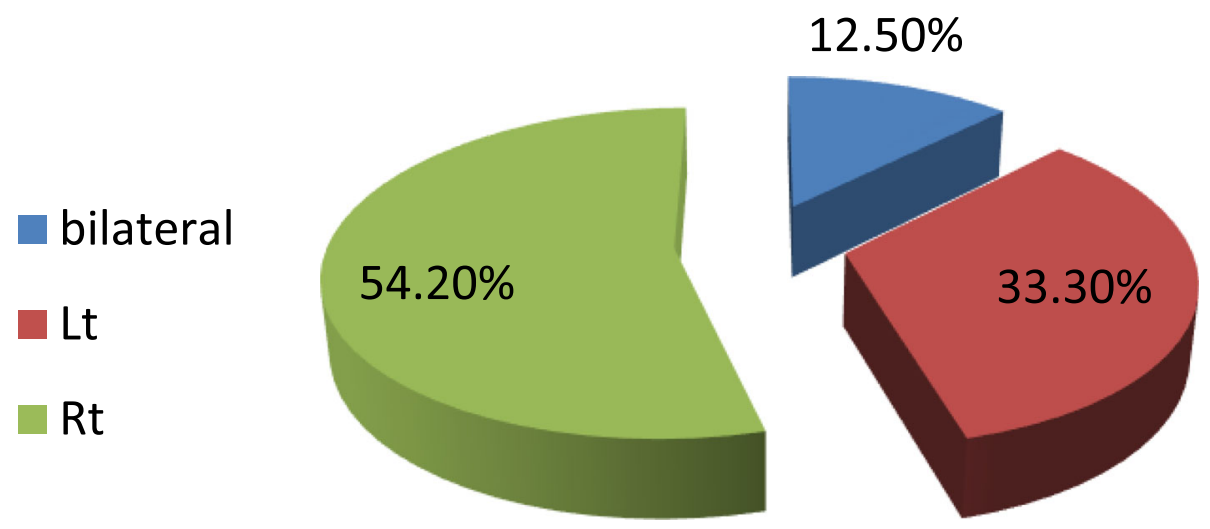

Fig. 5 Distribution of lesion sites among patients

Preferences of TLM over other modalities incorporate shorter hospitalization, lower morbidity, predominant valuable results, shorter treatment time, and less harm to sound tissue compared to those of RT. Also, an imperative advantage of utilizing TLM is that takes off all treatment choices open in patients with a recurrent tumor within a short period of follow-up [8].

Opposite to open surgical methods where dissection includes expelling anatomical structures, TLM is based on the real tumor spread itself, resecting only the included tissue and structures utilizing narrow margins to save uninvolved structures and so improve functional results [9].

This study included 33 patients with early-stage glottic carcinoma, who underwent transoral laser microsurgery (TLM).

Our aim in this study was to estimate the rate of recurrence after 24 months' follow-up.

In our study, the recurrence of the tumor occurred in 2 patients $(6.3 \%)$ and this in agreement with $\mathrm{Lu}$ et al. [10] as in this mentioned study 70 patients were followed up for 36 months and 6 patients (8.5\%) had a recurrence.

Zhang et al. also treated 32 patients with glottic cancer by TLM and the cases were followed up for 3 years [11].

During the follow-up period, granulation was found in all cases. Two patients treated by a secondary transoral

Table 2 Distribution of comorbidities among patient

\begin{tabular}{llll}
\hline & & Count & $\%$ \\
\hline Comorbidities & Free & 29 & $87.9 \%$ \\
& HCV & 1 & $3 \%$ \\
& Diabetic & 1 & $3 \%$ \\
& HTN & 2 & $6 \%$ \\
\hline
\end{tabular}

$\mathrm{CO} 2$ laser surgery and the other case had a total laryngectomy; all three cases were followed up for 5 years without recurrence.

But in our study, the follow-up duration was just 24 months contrary to Zhang et al. [11] and Lu et al. [10].

Rumeau et al. [12] treated 93 patients with early-stage glottic carcinomas (Tis/T2) by TLM.

The patients experienced fiberoptic endoscopy 6 weeks after surgery and a systematic second seen by direct laryngoscopy under general anesthesia at 3 months. Twenty patients had a local remaining disease or recurrence after the primary laser surgery, but 17 were rescued (85\%). Local residual disease and recurrence were more frequent in patients with progressed illness (T1b/ T2), invasion of the anterior commissure, and "non-safe" margins. In our study, we did not select patients with suspicious of cartilage invasion and all of our patients were $\mathrm{T}$ in situ or $\mathrm{T} 1 \mathrm{a} / \mathrm{T} 1 \mathrm{~b}$; for this reason, there was no high percentage of cases that need a second laser exposure as in Rumeau et al. [12].

An early laser excision strategy (e.g., inside the primary 2 months after TLM) or an alternative procedure may be talked about in this situation. "Watchful observation" should be saved for compliant patients only so that the chance of lost potential recurrences is minimized [12].

The duration of follow-up in Rumeau et al. [12] was short (3 months) in contrary to our study (24 months).

One patient $(3.03 \%)$ in our study was diagnosed with left glottic squamous cell carcinoma in situ and underwent TLM. After that, the patient experienced right glottic squamous cell carcinoma grade 2 after 5 months of follow-up.

Reinoso et al. [13] performed TLM on 58 patients diagnosed with squamous cell carcinoma of the glottic (T1a, T1b, T2). The study group included 57 (98.3\%) 


\section{follow up}

failure to remove whole
lesion \& refrred to
Radiotherapy

Free after 24 months

recurrent after 5 months

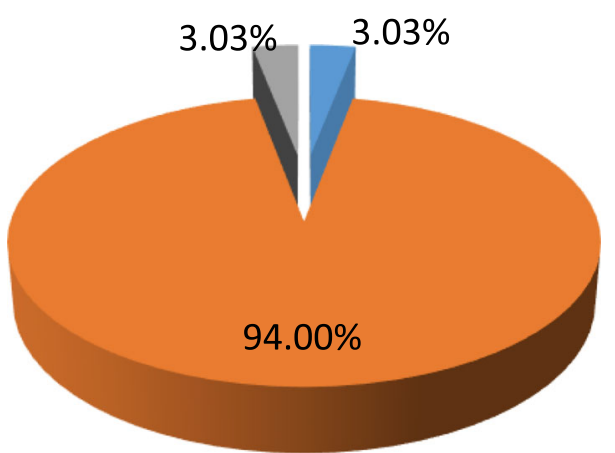

Fig. 6 Rate of recurrence in 24 months' follow-up

males and 1 (1.7\%) female. The mean age was 65.5 (min, $46 /$ max, 88).Over 3 years of follow-up, the recurrence rate had been $10.3 \%$ ( 6 patients).

Similarly, our study group included one female patient (3.03\%) and mean age was 60.5 ( $\min , 37 / \max , 80)$.

Amir and Vernham [14] reported a higher disease survival rate of about $93.3 \%$ at 18 months of follow-up when 30 patients (small number as in our study) underwent TLM.

A prospective cohort study by Song et al. [15] during 10 years identified a local control rate of $82 \%$ in 21 patients with T1b.

However, our study was a prospective clinical trial study.

Regarding the secondary outcomes of our study, the meantime of procedure was $17.75 \mathrm{~min}(\mathrm{~min}, 10 / \mathrm{max}$, 40 ) and the mean score of visual analog scale VAS of pain was 3.2 ( $\min , 2 / \max , 5)$.

These results are consistent with Shuang et al. [16] whose study was conducted on 186 patients with early glottic carcinoma to evaluate the voice and functional outcomes of $\mathrm{CO} 2$ laser. In the latter study, the mean time of the procedure was 12.8 and the mean score of visual analog scale VAS of pain was 2.8.

In a study by Bruzgielewicz et al. [17] among 55 patients, postoperative bleeding was the most commonly seen complication. All the cases of bleeding were easy to control. In 23 cases, the pain and mild laryngeal edema were observed and it resolved in 3-4 days after medical treatment.

Table 3 Secondary outcomes

\begin{tabular}{llllll}
\hline & Mean & $\begin{array}{l}\text { Standard } \\
\text { deviation }\end{array}$ & Median & Minimum & Maximum \\
\hline VAS pain scale & 3.42 & .72 & 3.00 & 2.00 & 5.00 \\
$\begin{array}{l}\text { Duration of } \\
\text { operation (min) }\end{array}$ & 17.75 & 7.12 & 15.00 & 10.00 & 40.00 \\
\hline
\end{tabular}

The VHI score results were not satisfied regarding voice outcome, and these results are in compatible with results of Kerr et al. [18] study when assessing the voice quality after laser and radiotherapy.

In our series, no significant complications were seen; no patient underwent tracheostomy and had symptoms of dyspnea, severe pain, hemoptysis, bleeding, surgical site infection, and other serious complications.

The limitation of our study is a relatively short duration of follow-up.

Larger sample size with longer follow-up period up to 5 years, including different advanced lesions and anterior commissure invasion, would further validate the findings of the study.

\section{Conclusion}

In carefully selected patients with laryngeal carcinomas, $\mathrm{CO}_{2}$ laser transoral microsurgery is an effective and reproducible single-stage modality therapy in patients with glottic carcinoma. Superficial close margins can be managed by a careful wait-and-see policy, while positive margins should undergo another surgical resection.

With appropriate surgical indication, detailed preoperative evaluation, and a good surgical skill, transoral $\mathrm{CO} 2$ laser surgery may achieve satisfactory outcome in the treatment of glottic cancer.

\section{Abbreviations}

TLM: Transoral laser micro-laryngeal; AJCC: American Joint Committee on Cancer; VAS: Visual analogue score; VHI: Voice Handicap Index; SPSS: Statistical Package for the Social Sciences; OPL: Open partial laryngectomy; RT: Radiotherapy

\section{Supplementary Information}

The online version contains supplementary material available at https://doi. org/10.1186/s43163-021-00107-8.

Additional file 1. Movie1 


\section{Acknowledgements}

Not applicable

\section{Authors' contributions}

AE was the main surgeon and gave us the idea of this study. MA was responsible for the data collection and writing of the manuscript. SB shared in revision of manuscript and assisted in the operations. AY shared in the data collection and revision of manuscript. All authors read and approved the final manuscript

\section{Funding}

No funding was obtained

\section{Availability of data and materials}

The datasets used or analyzed during the current study are available from the corresponding author on reasonable request.

\section{Declarations}

\section{Ethics approval and consent to participate}

The study was approved by scientific committee of ORL Department of Cairo University and ethics committee of Faculty of Medicine, Cairo University, but the committee reference number is not available. The informed written consent was obtained from all patients.

\section{Consent for publication}

Not applicable

\section{Competing interests}

The authors declare that they have no competing interests.

\section{Author details}

'Otolaryngology Department, Kasr Al-Aini, Faculty of Medicine, Cairo University, Cairo, Egypt. ${ }^{2}$ ENT Registrar, Queens Hospital, Romford, London, UK. ${ }^{3}$ Surgical Oncology, National Cancer Institute, Cairo University, Cairo, Egypt.

Received: 15 October 2020 Accepted: 11 April 2021

Published online: 07 May 2021

\section{References}

1. Ortholan C, Benezery K, Dassonville O (2012) A specific approach for elderly patients with head and neck cancer. Anti-Cancer Drugs 22(27):647-655

2. Steiner W, Ambrosch P, Braun U (2000) Endoscopic laser surgery of the upper aerodigestive tract: with special emphasis on cancer surgery. Clin Otolaryngol 34(4):367-373

3. Gleeson J, Clarke R (2008) Scott-Brown's Otorhinolaryngology: Head and Neck Surgery 7Ed: 3 volume set. CRC Press, New York. https://doi.org/10.12 01/b15118

4. National Comprehensive Cancer Network (NCCN) (2018) Head and neck cancers guidelines. TNM Staging:123-125

5. Mccormak H, Horne D, Sheather S (1988) Clinical applications of visual analogue scales: a critical review. Psychol Med 18(4):1007-1019. https://doi. org/10.1017/S0033291700009934

6. Jacobson B, Johnson A, Grywalski C, Silbergleit A, Jacobson G, Benninger M, Newman C (1997) The Voice Handicap Index (VHI): development and validation. Am J Speech Lang Pathol 6(3):66-70. https://doi.org/10.1044/1 058-0360.0603.66

7. Peretti G, Piazza C, Mora F, Garofolo S (2016) Reasonable limits for transoral laser microsurgery in laryngeal cancer. Otolaryngol Head Neck Surg 24(2): 135-139

8. Low T, Yeh D, Zhang T, Araslanova R, Hammond J, Palma D (2016) Evaluating organ preservation outcome as treatment end point for T1NO laryngeal cancer. Laryngoscope 127(6):1322-1327

9. Warner L, Chudasama J, Kelly C, Loughran S, McKnezie K, Wight R (2014) Radiotherapy versus open surgery versus endolaryngeal surgery with laser for earl laryngeal squamous cell carcinoma. Cochrane Database Syst Rv. Acta Otolaryngol 126(3):306-310

10. Lu Z, Song $X$, Zhang $\mathrm{S}$, Zhang $\mathrm{H}$, Chen $\mathrm{L}$ (2012) Long-term outcome of $\mathrm{CO}_{2}$ laser microlaryngoscopic treatment for laryngeal cancer. Zhonghua Zhong liu za zhi [Chinese Journal of Oncology] 34(6):473-476
11. Zhang, Tang, Chen (2017) Treatment of glottic cancer involving the anterior commissure by transoral CO2 laser surgery. Zhonghua Er Bi Yan Hou Tou Jing Wai Ke Za Zhi 52(5):337-342

12. Rumeau, Nguyen, Teixeira, Baumann \& Toussaint (2017): Watchful observation follow-up scheme after endoscopic CO2 laser treatment for small glottic carcinomas: A retrospective study. Department of ENT and head and neck surgery, University Hospital, Nancy, France. Clin Otolaryngol 2017:42(6):1193-1199

13. Reinoso F, Velasquez A, Fernandez J, Conde, Hidalgo (2016) Transoral CO2 laser microsurgery outcomes for early glottic carcinomas T1-T2. Otorhinolaryngol 20(3):212-217 Department of Otorhinolaryngology - Head and Neck Surgery, University Hospital of Vigo, Vigo, Pontevedra, Spain

14. Amir I, Vernham G (2015) Audit of transoral laser-assisted microsurgical resection of early laryngeal cancer. Clin Otolaryngol 34(4):367-373 Department of Otolaryngology and Head and Neck Surgery, NHS Lothian, Edinburgh, Scotland, UK

15. Song J, Rigby M, Trites J, Hart R, Taylor S (2017) Outcomes of transoral laser microsurgical management of T1b stage glottic cancer. J Laryngol Otol 131(5):433-441. https://doi.org/10.1017/50022215117000329

16. Yu S, Li C, Zhou X, Huang Y, Zhang L (2016) Outcomes of radiofrequency ablation (RFA) and CO2 laser for early glottic cancer. Am J Otolaryngol 37(4):311-316

17. Bruzgielewicz A, Osuch-Wójcikiewicz E, Januszek G, Szwedowicz P (2011) The complications after $\mathrm{CO} 2$ laser endoscopic surgery for early glottic cancer. J Laryngol Otol 115(4):298-301

18. Paul Kerr S, Taylor M, Rigby M (2012) Oncologic and voice outcomes after treatment of early glottic cancer: transoral laser microsurgery versus radiotherapy. J Otolaryngol Head Neck Surg 41(6):381-388

\section{Publisher's Note}

Springer Nature remains neutral with regard to jurisdictional claims in published maps and institutional affiliations.

\section{Submit your manuscript to a SpringerOpen ${ }^{\circ}$ journal and benefit from:}

- Convenient online submission

- Rigorous peer review

- Open access: articles freely available online

High visibility within the field

- Retaining the copyright to your article

Submit your next manuscript at $\boldsymbol{\nabla}$ springeropen.com 